\title{
Edge Computing for IoT-Enabled Smart Grid
}

\author{
M. Yasir Mehmood, ${ }^{1}$ Ammar Oad $\mathbb{D}^{2},{ }^{2}$ Muhammad Abrar, ${ }^{1}$ Hafiz Mudassir Munir $\mathbb{D},{ }^{3}$ \\ Syed Faraz Hasan, ${ }^{4}$ H. Abd ul Muqeet, ${ }^{5}$ and Noorbakhsh Amiri Golilarz ${ }^{6}$ \\ ${ }^{1}$ Department of Electrical Engineering, Bahauddin Zakariya University, Multan 60000, Pakistan \\ ${ }^{2}$ Faculty of Information Engineering, Shaoyang University, Shaoyang 422000, China \\ ${ }^{3}$ Department of Electrical Engineering, Sukkur IBA University, 65200 Sukkur, Pakistan \\ ${ }^{4}$ School of Engineering and Advanced Technology Massey University, Auckland 0632, New Zealand \\ ${ }^{5}$ Department of Electrical Engineering \& Technology, Punjab Tianjin University of Technology, Lahore, Pakistan \\ ${ }^{6}$ School of Computer Science and Engineering, University of Electronic Science and Technology of China, Chengdu 611731, China
}

Correspondence should be addressed to Ammar Oad; ammar.oad@hnsyu.edu.cn

Received 23 February 2021; Accepted 30 June 2021; Published 13 July 2021

Academic Editor: Flavio Lombardi

Copyright (c) 2021 M. Yasir Mehmood et al. This is an open access article distributed under the Creative Commons Attribution License, which permits unrestricted use, distribution, and reproduction in any medium, provided the original work is properly cited.

\begin{abstract}
Smart grid is a new vision of the conventional power grid to integrate green and renewable technologies. Smart grid (SG) has become a hot research topic with the development of new technologies, such as IoT, edge computing, artificial intelligence, big data, 5G, and so on. The efficiency of SG will be increased by smart embedded devices that have intelligent decision-making ability. Various types of sensors and data sources will collect data of high resolution. One of the vital challenges for IoT is to manage a large amount of data produced by sensors. Sending this massive amount of data directly to the cloud will create problems of latency, security, privacy, and high bandwidth utilization. This issue is addressed by edge computing (EC). In EC, the data are processed at the edge of the network that is near the embedded gadgets. This paper provides a comprehensive review of the smart grid systems, based on IoT and EC. The development in the rising technologies, the framework for EC-IoT-based SG, and requirements to implement the EC-IoT-based SG system are highlighted in the paper. Framework for EC-IoT-based SG is examined, and important requirements to implement the EC-IoT-based SG system are outlined. Finally, some critical issues and challenges faced in the implementation of EC-IoT-based SG systems are identified. Some important open research issues are also identified.
\end{abstract}

\section{Introduction}

An immense number of loosely interlinked synchronous alternate current grids are involved in traditional power grids. Generation, transmission, and distribution of electrical power are the three main activities of traditional grids [1]. The direction of power flow in this system is unidirectional. Electrical energy is generated by a large number of power plants like hydroelectric power plants, coal-fired power plants, nuclear power plants, and diesel-fired power plants. Then, the produced electricity is transmitted through high-voltage transmission lines to distant load centres. At the distribution end, the electrical power is distributed to consumers by the electrical distribution systems at a lower voltage. A centralized system is used to monitor and control each grid so that the energy generated by the power plants should be sufficient for the needs of consumers and within the limits of the power plants. All these tasks (generation, transmission, and distribution) are performed by utility companies, who supply electrical power to consumers, and a billing mechanism is used for the recovery of their costs and earning profit. The consumption of electrical energy has increased dramatically since 1970 [2]. A notable amount of energy is wasted because of ineffectual appliances of consumers, smart technology absence, irregular monitoring, communication, and system's deficiency to stock produced electrical energy [3]. Nowadays, power grids are facing some challenges like security, reliability, growing renewable energy sources, and rising energy demands.

The smart grid is an optimal solution to these challenges. There are numerous types of sensors and power terminals in smart grids like temperature sensors, humidity sensors, 
video sensors, current leakage sensors, vibration sensors, and humidity sensors. These are supported by intelligent power systems based on IoT [4]. One of the wide-ranging applications of IoT is the smart grid [5]. SG has a bidirectional information flow between consumers and service providers. Smart grid is equipped with unofficial usage detection, smart meters, advanced metering infrastructure (AMI), load balancing, and fault tolerance [6-8].

According to the need for energy, SG makes important decisions like electrical energy consumption scheduling, real-time pricing, self-healing, and power usage. The power quality and the efficiency of the grid can be improved by these decisions [8]. Automation, connectivity, and tracking of an immense number of devices require analysis, distributed monitoring, two-way communications, and highspeed control. These are the main objectives of the smart grid. Distributed automation of the smart grid is required to achieve these objectives.

IoT is a mesh of physical things or objects that are connected to the Internet. These objects interconnect with external and internal environments with the help of embedded technology. Such physical things analyze, sense, control, and decide independently or in alliance with other things by way of two-way communication and high-speed control. This is also essential for the smart grid [9]. IoT is the result of current progress in embedded processing, wireless, and sensing technologies. IoT offers improved and structured control and monitoring services [10]. Applications like smart home, health care, smart building, smart grid, smart industry, smart city, and smart agriculture are covered by IoT [11]. The number of objects connected to the Internet exceeded the number of people present in the world [1]. IoTbased smart grids need six fundamental technologies which include software-defined objects, model protocols, edge computing-based analysis, intelligent sensing, low cost, and network information security. Edge computing is the major technology to attain real-time demand response for IoTbased smart grids [12, 13].

IoT devices are generating an enormous amount of data, while the resources of IoT devices are limited. Therefore, the application tasks from the IoT devices should be offloaded to the remote cloud data centres. If all the tasks are offloaded to the cloud, then it puts a significant burden on the network. This issue can be solved by edge computing. Computation offloading is formulated as an optimization problem to minimize offloading cost and provide performance guarantees [14-19]. Cloud computing resources are located at distant data centres due to which communication latency and network bandwidth become serious problems. For emerging IoT applications, EC is a better choice than cloud because computing resources are available closer to the user. Our survey deals with the integration of edge computing with IoT-based smart grid. Framework for EC-IoT-based SG is examined, and important requirements to implement the EC-IoT-based SG system are outlined. Finally, some critical issues and challenges faced in the implementation of ECIoT-based SG systems are also identified.

This paper is organized as follows. In Section 2, surveys related to EC and IoT-based SG are provided. Section 3 provides an overview of IoT and EC. In this section, the development of key technologies in the context of the IoT domain is presented and EC is compared with fog computing and cloud computing. Section 4 reviews IoT-enabled SG. The integration of EC with IoT-enabled SG is discussed in Section 5 , in which the framework for EC-IoT-based SG is presented and some important requirements to implement this framework are discussed. Open research challenges and issues are provided in Section 6. The paper is concluded in Section 7.

\section{Related Work}

In this section, the literature reviews of the related terminologies such as edge computing and IoT-based smart grid are discussed.

2.1. Edge Computing. EC is classified into different groups based on architecture. Their performance is compared by bandwidth, network latency, integrity, availability, overhead, and energy consumption [20]. Research on EC and technologies implementing EC are reviewed in [21]. Reference [22] investigates the principal challenges of mobile edge computing (MEC) related to wireless resources that are based on evolutionary and classic games. The utilization of EC technology to implement IoT applications is surveyed in [23]. The technical aspects of permitting MEC into IoT are also discussed, and some insights into other integration technologies are also provided. Shahzadi et al. [24] provided a review of widespread MEC frameworks together with relative analysis of present approaches concerning different performance parameters. The relative analysis uses different parameters such as network performance, system performance, system migration overhead, and deployment overhead to estimate the usefulness of various approaches. Future research directions and state of the art for multiaccess edge computing are also investigated.

In [25], a tutorial is presented on three models of EC technologies including fog computing, cloudlets, and MEC. Architectures, principles, applications, efforts, and standardization are outlined and compared in this tutorial. The characteristics of fog computing based on radio access networks and the difference between fog computing and mobile edge computing in terms of radio access networks are also discussed. First, an overview on mobile edge networks including architecture, definition, and advantages is given and then a comprehensive review on communication techniques, computing, and caching at the edge of the network is presented in [26]. The use cases and applications of edge network are discussed, and future directions and open research challenges are also indicated. In [27], Mao et al. mainly focused on MEC research with the centre of attention joint radio and computational resource management. The main objective of [28] is to analyze challenges, security threats, and operations in edge paradigms. Venues of collaboration and potential cooperation are highlighted.

Afolabi et al. [29] described the slicing of the network concerning an end-to-end viewpoint. Key concepts, 
historical inheritance, solutions, and enabling technologies are also elaborated. More specifically, the work in [29] reviews the network needs and different use cases of network slicing, end-to-end arrangement and operation, preslicing period, radio access encompassing, core network, and transport network. The details of particular slicing solutions for every segment of the $5 \mathrm{G}$ system are also provided. The terms including cloudlets, fog computing, and MEC are investigated together under the super ordinate concept of "edge computing" [30]. The real potential of EC can be achieved by serving the SDN as an enabler to lessen the complexity barriers. A review of the MEC framework and architecture defined by ETSI and tutorial on MEC technology is presented in [31]. The reference scenarios and vital use cases where MEC can be applied are first described and then present technologies integrating MEC features to the mobile networks are surveyed, and present development in MEC standardization is discussed in [32]. Some examples of MEC placement with particular reference to IoT cases are also provided. Edge-centric vision enfolding and its future research challenges are the main objectives of [33].

None of the surveys presented above focus on the application of EC in SG. Table 1 presents the summary of the above surveys which also supports our argument. We intend to present a comprehensive review that covers the application of EC in SG.

2.2. IoT-Based Smart Grid. Turjman and Abujubbeh [34] focused on the comprehensive review of the feasibility of implementing smart meter for reliability and power quality monitoring. An overview is provided on wireless communication technologies, routing algorithms, and smart meters as enabling technologies in advanced meter infrastructure (AMI). Another survey [35] provides a short review of technologies and tools that are used in smart electricity meter data analytics. Similarly, Parra et al. [36] focused on a security tool for SG and industrial Internet of things (IIoT) named deep packet inspection. Existing models and recommendations are studied and deep packet inspection is evaluated as a security tool for smart grid. The main focus is security threats and vulnerabilities in IoT-based SG systems.

The application of IoT in electrical power and energy systems is studied in [37]. The IoT value and significance in a network of electrical power, challenges, recommendations, and environmental, economic, and collective effects of electrical power systems are investigated. In [37], the authors provide a review on smart grid technologies. It is a short survey that also includes Io $\mathrm{T}$ and energy management system as well as smart metering. The application of IoT in SG is presented in [38], and a short overview of challenges, opportunities, and future directions is also provided. Sun et al. [39] provided a systematized review on evolution and deployment of smart meters; the authors also presented recommendations regarding future evolution of smart energy meters. Reference [40] aims to present a comprehensive survey on the application of blockchain in SG, and important security challenges that can be directed by blockchain are identified. Several present works addressing security issues in the domain of SG by blockchain are presented, and important research challenges and future research directions of the application of blockchain in security issues of the smart grid are also discussed. The framework for SG communication's predicted capabilities and capacities is presented in [41]. The studies and solutions in which $5 \mathrm{G}$ is used to empower the applicability of smart grids are surveyed. The importance of a key management system (KMS) for the security of advanced metering infrastructure (AMI) in the smart grid is highlighted in, [42]. The survey presents all the important security challenges of AMI in SG. First, the installation framework of SG, the major characteristics of AMIs, and the interconnection between AMI and SG are discussed. Then, main features of AMI are explored and security challenges and issues are introduced; finally, the challenges and future directions for KMS in AMI are summarized. Musleh et al. [43] surveyed the history, diversity, and consequences of the "False Data Injection Attacks (FDIAs)" in SG so that improved recognition algorithms could be developed to address these challenges; different techniques are used for the detection of FDIA in smart grids; the criteria for most important growing future FDIA recognition algorithms is defined under the considerations of NIST's guidelines. A short overview of real cyberattacks on legacy energy networks and the smart metering networks is provided in [44]; various schemes are proposed to handle these attacks, and future research directions in smart grid metering networks are also investigated. Another survey [45] discusses present constraints in various microgrid configurations that relate to particular communication performance metrics with the successful functioning of microgrid with wireless and wired technologies. The impact of wired and wireless communications on the microgrid is experimentally analyzed. The survey aims to pinpoint the requirements of communication networks for solid applications of MGs and MG clusters. Reference [46] discusses the alternatives of XMPP as middleware and web services and the replacement of XML for serialization. YAM14, ICE, and ZeroMQ are the middleware that works well, and protostuff and protobuff are the serializations that will perform better. A comprehensive review of IoT-aided SG systems is presented in [47]. It consists of present architectures, implementations, and prototypes of IoT-aided SG systems. The summary of the above surveys is comparatively presented in Table 2 . IoT and EC are the peer technologies for the future SG. The following section provides a brief overview of these technologies.

\section{IoT and Edge Computing: An Overview}

In this section, Internet of things is described to explore its various aspects.

3.1. Internet of Things. IoT is the structure of interconnected devices that can transfer data over the network, where it is interpreted on the computer by humans. Millions of smart devices are established in composite networks to deliver energetic functionalities which include monitoring and 
TABLE 1: Summary of surveys related to MEC (communication technology (CT), smart meter (SM), advanced metering infrastructure (AMI), and power quality (PQ)).

\begin{tabular}{|c|c|c|c|c|c|c|c|c|}
\hline Reference & Main objective & $\begin{array}{c}\text { Smart } \\
\text { grid }\end{array}$ & IoT & MEC & $\begin{array}{l}\text { Fog } \\
\text { computing }\end{array}$ & Cloudlet & $\begin{array}{l}\text { Evolution of } \\
\text { edge } \\
\text { computing }\end{array}$ & $\begin{array}{l}\text { Edge } \\
\text { computing } \\
\text { networking }\end{array}$ \\
\hline $\begin{array}{l}\text { Our } \\
\text { survey }\end{array}$ & $\begin{array}{c}\text { The integration of edge computing with } \\
\text { smart grid }\end{array}$ & $\checkmark$ & $\checkmark$ & $\checkmark$ & $\checkmark$ & $\checkmark$ & $\checkmark$ & $\checkmark$ \\
\hline$[20]$ & Classification of edge computing & $x$ & $\sqrt{ }$ & $\sqrt{ }$ & $x$ & $x$ & $\checkmark$ & $x$ \\
\hline [21] & $\begin{array}{l}\text { Research on MEC and technologies } \\
\text { implementing edge computing }\end{array}$ & $x$ & $\checkmark$ & $\checkmark$ & $x$ & & $\checkmark$ & $\checkmark$ \\
\hline$[22]$ & Game theory and edge network structure & $x$ & $x$ & $\checkmark$ & $\checkmark$ & $x$ & $x$ & $\checkmark$ \\
\hline [23] & Edge computing in IoT applications & $x$ & $\sqrt{ }$ & $\checkmark$ & $x$ & $x$ & $\checkmark$ & $\checkmark$ \\
\hline$[24]$ & $\begin{array}{l}\text { A review on the state of the art and future } \\
\text { research directions on MEC }\end{array}$ & $x$ & $\checkmark$ & $\checkmark$ & $\checkmark$ & $\checkmark$ & $\checkmark$ & $\checkmark$ \\
\hline [25] & $\begin{array}{l}\text { A tutorial on three-model edge computing } \\
\text { technologies including cloudlets, fog } \\
\text { computing, and MEC }\end{array}$ & $x$ & $\checkmark$ & $\checkmark$ & $\checkmark$ & $\checkmark$ & $\checkmark$ & $x$ \\
\hline [26] & $\begin{array}{c}\text { A comprehensive review of issues on } \\
\text { communication techniques, computing, and } \\
\text { caching in MEC }\end{array}$ & $x$ & $x$ & $\checkmark$ & $\checkmark$ & $\checkmark$ & $\checkmark$ & $\checkmark$ \\
\hline [27] & $\begin{array}{c}\text { An investigation on joint radio and } \\
\text { computational resource management in } \\
\text { MEC }\end{array}$ & $x$ & $x$ & $\checkmark$ & $x$ & $x$ & $\checkmark$ & $\checkmark$ \\
\hline [28] & $\begin{array}{c}\text { To analyze challenges, security threats, and } \\
\text { operations in edge paradigms }\end{array}$ & $x$ & $x$ & $\checkmark$ & $\checkmark$ & $x$ & $\checkmark$ & $x$ \\
\hline [29] & $\begin{array}{l}\text { Description of network slicing from an E2E } \\
\text { perspective on enabling technologies, } \\
\text { principles, and solutions }\end{array}$ & $x$ & $x$ & $\checkmark$ & $x$ & $x$ & $x$ & $x$ \\
\hline [30] & $\begin{array}{l}\text { A review on SDN for enabling edge } \\
\text { computing, use cases, and future directions } \\
\text { A review of mobile edge computing }\end{array}$ & $x$ & $\checkmark$ & $\checkmark$ & $\checkmark$ & $\checkmark$ & $\checkmark$ & $\checkmark$ \\
\hline [31] & $\begin{array}{l}\text { framework and architecture defined by ETSI } \\
\text { and tutorial on MEC technology }\end{array}$ & $x$ & $\checkmark$ & $\checkmark$ & $x$ & $x$ & $\checkmark$ & $x$ \\
\hline [32] & $\begin{array}{l}\text { A comprehensive survey on computation } \\
\text { offloading, computing resources allocation, } \\
\text { and mobility management and discussion of } \\
\text { vital use cases and standardization of MEC }\end{array}$ & $x$ & $x$ & $\checkmark$ & $x$ & $x$ & $\checkmark$ & $x$ \\
\hline [33] & $\begin{array}{l}\text { A description of edge-centric vision and } \\
\text { future research challenges }\end{array}$ & $x$ & $x$ & $\checkmark$ & $x$ & $x$ & $\checkmark$ & $x$ \\
\hline
\end{tabular}

TABLE 2: Summary of surveys related to IoT-based SG.

\begin{tabular}{|c|c|c|c|c|c|c|c|c|c|c|}
\hline Reference & Year of publication & Smart grid & IoT & $\mathrm{CT}$ & SM & AMI & Security & PQ & Architecture & Blockchain \\
\hline [34] & 2019 & $\sqrt{ }$ & $\sqrt{ }$ & $\checkmark$ & $\sqrt{ }$ & $\sqrt{ }$ & $\sqrt{ }$ & $\checkmark$ & $\sqrt{ }$ & $\sqrt{ }$ \\
\hline$[35]$ & 2017 & $\checkmark$ & $x$ & $x$ & $\checkmark$ & $\checkmark$ & $\checkmark$ & $\checkmark$ & $x$ & $x$ \\
\hline$[36]$ & 2019 & $\checkmark$ & $\checkmark$ & $\sqrt{ }$ & $x$ & $\checkmark$ & $\checkmark$ & $x$ & $x$ & $x$ \\
\hline [48] & 2019 & $\checkmark$ & $\checkmark$ & $\checkmark$ & $x$ & $x$ & $\checkmark$ & $x$ & $\checkmark$ & $x$ \\
\hline [37] & 2018 & $\checkmark$ & $\checkmark$ & $\checkmark$ & $x$ & $x$ & $\checkmark$ & $x$ & $\checkmark$ & $x$ \\
\hline$[38]$ & 2014 & $\checkmark$ & $\checkmark$ & $\checkmark$ & $\checkmark$ & $\checkmark$ & $x$ & $x$ & $\checkmark$ & $x$ \\
\hline [39] & 2015 & $\checkmark$ & $x$ & $x$ & $\checkmark$ & $x$ & $x$ & $x$ & $x$ & $x$ \\
\hline [40] & 2020 & $\checkmark$ & $x$ & $x$ & $x$ & $x$ & & $x$ & $x$ & \\
\hline [41] & 2019 & $\checkmark$ & $x$ & $\checkmark$ & $x$ & $x$ & $x$ & $x$ & $x$ & $x$ \\
\hline [42] & 2018 & $\checkmark$ & $x$ & $x$ & $\checkmark$ & $\checkmark$ & $\checkmark$ & $x$ & $x$ & $x$ \\
\hline [43] & 2020 & $\checkmark$ & $x$ & $x$ & $x$ & $x$ & $\checkmark$ & $x$ & $x$ & $x$ \\
\hline [44] & 2019 & $\checkmark$ & $x$ & $\checkmark$ & $\checkmark$ & $\checkmark$ & $\checkmark$ & $\checkmark$ & $x$ & $x$ \\
\hline [45] & 2020 & $\checkmark$ & $x$ & $\checkmark$ & $x$ & $x$ & $\checkmark$ & $x$ & $\checkmark$ & $x$ \\
\hline [46] & 2017 & $\checkmark$ & $\checkmark$ & $\checkmark$ & $x$ & $x$ & $x$ & $x$ & $x$ & $x$ \\
\hline [47] & 2019 & $\checkmark$ & $\checkmark$ & $\checkmark$ & $\checkmark$ & $\checkmark$ & $x$ & $x$ & $x$ & $x$ \\
\hline
\end{tabular}


controlling important infrastructure and communications [49]. Three main concepts are focused on IoT, things-oriented, internet-oriented, and semantic-oriented. Smart devices like sensors, RFID tags, actuators, laser scanners, cameras, NFC, and GPS are involved in things-oriented concepts. The internet-oriented concept provides communication between smart devices by means of different communication technologies like WiFi, ZigBee, Bluetooth, and so on.

The semantic-oriented concept comprehends diverse applications with the assistance of smart devices. The importance of IoT technology has increased in recent years. IoT interconnects different network embedded devices that are used in daily life with the Internet. Various systems like military, healthcare, home appliances, transportation, surveillance, security, power grids, and agriculture have been automated with the help of IoT [50]. Almost every feature of IoT has been investigated in literature reviews [51-54] beginning from architectural elements and the essential permissive technologies to the field of implementation and open challenges.

The wireless networks that are used for communications in IoT are investigated in [55-60]. IoT objects are standard devices that are equipped with transceivers, protocol stacks, and microcontroller. Figure 1 shows the Internet of things and connected components. Completely automated systems can be realized by enabling communication of IoT devices with other devices and with external entities like humans [61]. The emerging and present technologies for the health Internet of things are reviewed in [62].

The development of health Internet of things is underlined by three major technologies: (1) sensing, where there is an escalated push in power efficiency and miniaturization; (2) communications, where pervasive connectivity, wide availability of cloud infrastructure and standardized protocols are enabling factors; and (3) inference and data analytics, where the accessibility of massive amount of data and computational resources is transforming algorithms for characterizing inference and actions in health management. The physical Internet (PI or $\pi$ ) [63] has evolved as a worldwide logistics system where products are moved, stored, and handled efficiently and sustainably. To accomplish this goal, PI needs a high-level interconnectivity between the physical, operational, and informational features, enabled by an interconnected network of collaborative protocols, intermodel hubs and smart, modular, as well as standardized containers. In this frame of reference, $\mathrm{PI}$ is the vital contestant to benefit from the IoT revolution as it substantially provides end-to-end visibility of PI objects, systems, and operations through universal information exchange. Figure 2 shows the development of key technologies in the context of the IoT application domain.

3.2. Edge Computing. With the speedy evolution of the Internet of things, the number of smart devices connected to the Internet is increasing day by day, which has resulted in the unavailability of bandwidth, poor privacy, poor security, and low response in conventional cloud computing paradigms. To solve these issues, a new computing paradigm named "edge" has been introduced.

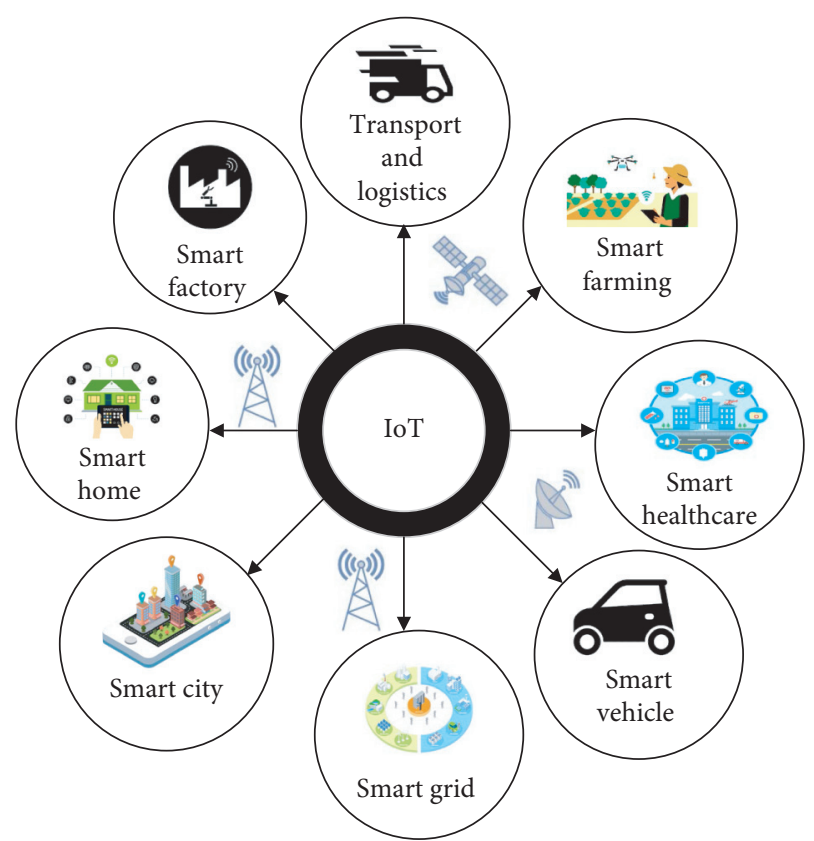

Figure 1: Internet of things.

The development of edge computing is introduced in [64]-[66]. Weisong et al. [64] defines EC as any network and computing resources through the path between data sources and cloud data centers. The authors sketch the framework of EC in which cloud service is represented by EC downlink data, the network and computing resources among the origin of data and the pathway of cloud computing center are represented by the edge of EC and the IoT is illustrated by the uplink data. A professor at "Carnegie Mellon University" in the US named Satya Narayanan sketches edge computing as "edge computing is a new computing model that deploys computing and storage resources (such as cloudlets, microdata centres, or fog notes) at the edge of the network closer to the mobile devices or sensors" [67]. EC carries out computation at the edge of the network. It emphasizes near to the user and near to the data source. It is real-time, reliable, and faster. The problems such as excessive energy utilization by cloud computing, pressure on network bandwidth, time delay, and higher costs of cloud infrastructure can be solved by this paradigm [68]. EC can fulfil the crucial requirements of the industry related to IT in optimization of data, sharp linking, application surveillance, real-time business, privacy, and security. Nowadays, EC is the centre of research for researchers [69-73].

\subsubsection{Difference between Edge and Fog. Fog computing is} defined by SISCO as "a highly virtualized platform that provides computing, storage, and networking services between end devices and traditional cloud computing data centres, typically but not exclusively located at the edge of the network" [74]. Fog computing provides networking, computing, and storage facilities among end-user devices and conventional cloud computing centres, and it is a supremely virtualized platform. It is generally understood as execution of edge computing $[28,75,76]$. The function of fog 


\begin{tabular}{|c|c|}
\hline \multicolumn{2}{|r|}{ Home and personnel } \\
\hline (i) & $\begin{array}{l}\text { Worldwide addressing } \\
\text { schemes like IPV6 }\end{array}$ \\
\hline (ii) & $\begin{array}{l}\text { Low power } \\
\text { consumption and slim } \\
\text { batteries }\end{array}$ \\
\hline (iii) & Cloud storage \\
\hline (iv) & $\begin{array}{l}\text { Standardization for } \\
\text { interoperability }\end{array}$ \\
\hline (v) & $\begin{array}{l}\text { RFID and cloud } \\
\text { security }\end{array}$ \\
\hline
\end{tabular}

(i) Data centres

(ii) Smart sensors in healthcare

\begin{tabular}{|l|}
\hline \multicolumn{1}{|c|}{ Business and industry } \\
\hline (i) Network sensors \\
(ii) Smart antennas \\
(iii) Increased RFID privacy \\
and security \\
(iv) Cloud computation as \\
a service \\
(v) Energy harvesting \\
(vi) Wireless power \\
(vii) Intelligent analytics \\
(viii) Interoperability \\
between WSNs and RFID \\
(ix) Miniaturized reader \\
\end{tabular}

(i) Industrial ecosystems

(ii) RFID in retail

(iii) Low-cost SCADA systems

\begin{tabular}{|c|c|}
\hline & Utility \\
\hline (i) & $\begin{array}{l}\text { Energy harvesting and } \\
\text { recycling }\end{array}$ \\
\hline (ii) & $\begin{array}{l}\text { Large-scale wireless } \\
\text { sensor networks }\end{array}$ \\
\hline (iii) & Self-adaptive system \\
\hline (iv) & $\begin{array}{l}\text { Deploy and forget } \\
\text { netwoks }\end{array}$ \\
\hline (v) & $\begin{array}{l}\text { Highly enhanced } \\
\text { security }\end{array}$ \\
\hline (vi) & $\begin{array}{l}\text { Cloud storage, } \\
\text { computation, and } \\
\text { online analytics }\end{array}$ \\
\hline
\end{tabular}

(i) Critical infrastrtucture monitoring

(ii) Smart grid and household metering

\begin{tabular}{|l|}
\hline Transport and logistics \\
(i) Smart grid tags for \\
vehicles and logistics \\
management \\
(ii) Automated vehicles \\
using IoT \\
(iii) System-level analytics \\
(iv) Diverse systems with \\
interaction between \\
other subnetworks \\
(v) Vehicle to \\
infrastructure \\
(i) Automated vehicles \\
(ii) Smart traffic \\
(iii) Intelligent transportation \\
and logistics
\end{tabular}

FIgURE 2: The development of key technologies in the context of IoT application domain.

TABle 3: Comparison of edge and fog.

\begin{tabular}{lcc}
\hline Features & Edge computing & Fog computing \\
\hline Network latency & Very low & Low \\
Overhead server & Very low & Low \\
Network access & LAN or WLAN & LAN \\
Response time & High & Slightly low \\
Energy utilization & Less & Less \\
Server scalability & High and disperse & Low and disperse \\
Reliable & Low & High \\
Standardization & No & Yes \\
Distribution & Largely distributed & Distributed \\
Storage & Low & Low \\
\hline
\end{tabular}

computing is to provide storage, disperse computing, networking potentialities, and control near to the user [77]. Table 3 provides a comparison between fog computing and edge computing.

3.2.2. Comparison of Edge and Cloud. Cloud computing is defined in NIST [78] as "a model for enabling ubiquitous, convenient, on-demand network access to a shared pool of configurable computing resources (e.g., networks, servers, storage, application, and services) that can be rapidly provisioned and released with minimal management effort or service provider interaction." The authors in [79] proposed a reference architecture for cloud computing. It presents a primal overview and recognizes the major participants and their responsibilities in cloud computing. Cloud computing consists of two-layer architecture (end device and data centre of cloud) while edge computing may have three-, four-, or five-layer architecture. In the edge paradigm, networking, storage, and computation are performed near to the end user and also in the central data centre. In cloud computing, entire data processing is in central data centre and end devices should hold up from performing subsequent step until the processed results of data are returned by the data centre. Storage and computation in EC is performed at various sites because of the accessibility of various levels of processing capacity dispersed in multi-layer infrastructures. To highlight the differences between edge computing and cloud computing, Table 4 sums up their differences regarding their technical parameters and general characteristics $[28,68,80,81]$.

IoT is the driving force behind the SG concept. It plays an important role in grid construction and improves maintenance, planning, and operations in SG. The following section discusses the integration of IoT with SG.

\section{IoT-Enabled Smart Grid}

The IoT technology offers a remarkable role in the construction of the grid. The main objective of the smart grid is to enhance planning, operations, and maintenance by verifying that every component of the grid is capable of "listening" and "talking." If there is any disruption in the service, then it is known by the utility company only when the customer complains about that disruption. In SG, the disconnection of service will be automatically known by the utility because particular modules of the smart grid-like smart meters will send the collected data from the sensors. In this scenario, the IoT plays an important role because each component in the smart grid will have an IP address and it should support two-way communication. This is achieved with the help of IoT. Cooperation is required for the realization of high-speed, real-time, and two-way sharing of data across different applications [82]. The implementation of IoT in smart grids is categorized into three types, predicted on IoT three-layered architecture [83, 84]. First, IoT is implemented for the deployment of different smart devices that monitor the states of various components of SG (at IoT 
TABLE 4: Comparison of edge and cloud.

\begin{tabular}{lcc}
\hline Parameters & Edge computing & Cloud computing \\
\hline Application locality & Local & Global \\
Real-time & High & Low \\
Network access & LAN or WLAN & LAN or WLAN \\
Availability & Highly redundant & High \\
Content generator & Humans, sensors, and devices & Mostly humans \\
Generation of content & Anywhere & Central \\
Latency & Low & High \\
Mode of control & Distributed & Centralized \\
Mobility support & Supported & Limited \\
Service access & At the edge & Centralized \\
Distance among server and client & One or several hops & Several hops \\
Server nodes & Very large & Few \\
Pressure of network bandwidth & Low & High \\
Location awareness & Yes & No \\
Reliable & Low & High \\
Standardization & No & Yes \\
Storage & Low & High \\
\hline
\end{tabular}

perception layer). Second, with the help of IoT smart devices, information is collected from the equipment through different communication technologies (at the network layer of IoT). Third, the IoT is implemented to control the SG by application interfaces (at the IoT application layer). The sensing devices of IoT generally consist of RFIDs, wireless sensors, cameras, machine-e-machine devices, infrared sensors, GPS, and laser scanners. SG information sensing is highly improved and supported by IoT technology. The IoT technology contributes a lot to the deployment of transmission and data sensing infrastructure for the SG, and it assists in network construction, maintenance, operation, security monitoring, safety management, user interaction, information collection, measurement, etc.

Moreover, the integration of power flow, distribution flow, and information flow in the SG is also enabled by IoT [85]. The main focus of existing SG architectures is to fulfil the necessities of power distributors to control the entire power grid [86]. A network of smart meters is used to access the consumers with the help of the "General Packet Radio Service (GPRS)."

A smart grid consists of four major subsystems, power generation, transmission, distribution, and power utilization. The application of IoT circles all these subsystems. In the power generation domain, IoT can be applied to control and monitor the energy consumption, equipment, units, pollutants' discharge, gas emissions, power connection, and energy storage, as well as for the management of distributed power plants, wind power, pumped storage, photo-voltaic, and biomass power plants $[87,88]$. In power transmission, IoT can be applied for controlling and monitoring transmission lines and substations and protection of transmission tower. In power distribution, IoT can be applied for distribution automation and equipment and operation management. In the power utilization domain, the IoT can be applied for automated meter reading, smart homes, charging and discharging of electric vehicles, energy consumption, home appliance information, controlling of power load, management and monitoring of energy efficiency, and management of power demand.
4.1. Smart Grid Communication Networks. There are three communication network layers of SG in terms of IoT technology and communication technologies: (a) home area networks (HANs), (b) neighbor area networks (NANs), and (c) wide area networks (WANs) [89].

4.1.1. Home Area Networks. HANs control the on-demand energy requirements of the consumers and comprise home appliances (air conditioners, televisions, washing machines, ovens, and refrigerators), smart devices, electrical vehicles, and renewable energy resources. It is deployed in commercial buildings, industrial plants, and residential units, and it provides the connection between electrical appliances and smart meters [2]. The communication technologies that are preferably used in HANs are WiFi, Bluetooth, ZigBee (Wireless technologies), and power line communications (wired technology). Wireless technologies are more suitable for HANs because their configuration is simple and nodes are excessive. The HAN uses the signal that has lower power with a short data range and lower frequencies [90]. Generally, there are three major components of HAN: gateways, communication protocols, and devices. All the devices communicate with each other through gateways. The compatibility between hardware devices and the exchange of data among them is ensured by the communication protocol. The devices are utilized to deliver information for the aim of energy management [91]. The function of IoT and advance sensing for the monitoring of energy flow in the smart grid is presented in [92].

4.1.2. Neighbor Area Networks. NANs are deployed in the distribution domain [93]. It is the second communication network layer of SG and contains smart meters that belong to numerous HANs. NAN facilitates communication among field devices and distribution substations. It gathers metering and service information and then it sends this information to data collectors that connect the NANs to the WAN [2]. 
The main purpose of NAN in the SG is to perform as a communication bridge, linking HAN to WAN, and through this, real-time energy consumption data of smart appliances placed in HAN are transmitted [94]. Both wired and wireless communication technologies are suitable for NAN. The ability of $5 \mathrm{G}$ and optical fibre to handle real-time latency applications makes them supreme technologies for NAN applications. NAN is largely deployed in AMI, and its range of application areas is expanding, e.g., distributed energy resource integration and advanced distribution automation [95]. In [96], the authors provide a structured approach to produce a scale-free topology for wireless-mesh NANs in SG. To confirm the scale-free property generated from the proposed approach, theoretical analysis is performed. Reference [97] presents a "constrained broadcast scheme with minimized latency (CBS-ML)" for NAN communications. The broadcast is constrained to a less number of core nodes to keep away from traffic congestion.

4.1.3. Wide Area Networks. WAN is considered as a backbone network in the power system as it provides the connection between the major core network and widely expanded smaller networks (NAN, IAN, and BAN). It provides unification between power transmission systems, renewable energy sources, bulk generation systems, and control systems [2]. PMUs that are deployed on the bus bar of the substation record the voltage and current phasor data. The data obtained from these PMUs are sent to the territorial transmission operator via WAN. The energy management system makes use of these data for protection, monitoring, and real-time control of the grid [98]. High bandwidth is required by WAN to transmit data from the backhaul network to the principal control centre. So, the communication technologies that are used for WAN generally have larger bandwidth, high data rate, low latency, and the largest range. Both wired and wireless technologies can be utilized in WAN connectivity. Fibre optics and PLC provide the efficient and secure transfer of data; however, cellular technologies are preferred by more utility vendors to connect WAN to the AMI system [99]. Cellular technologies are efficient and fast WAN connectivity solutions.

4.2. IoT-Enabled SG Architectures. Several architectures are proposed for IoT-based smart grid. A three-layer architecture is extensively used in the literature for IoT-based SG systems. The three-layer architecture proposed in [100-105] is comprised of three layers, perception layer, network layer, and application layer. The perception layer utilizes various types of sensors, tags, and readers to collect data from devices or terminals. It is also called the device layer. The network layer consists of various kinds of wired and wireless communication networks (like WiFi, ZigBee, 2G, 3G, 4G, cable, broadband, and private networks) and the Internet. Due to its mature technologies, the network layer is widely accepted. It maps the information collected by the sensors in the perception layer to the communication protocols. These mapped data are routed and transmitted to the application layer. The IoT information and management centres are also related to the network layer. The application layer processes the information obtained from the network layer and then it troubleshoots and monitors the IoT devices in real time, based on the processed information. It makes use of various types of IoT technologies to handle a wide set of IoT applications and has an application structure.

Application structure manages the computing, information processing, and interface with resources. The integration of communication technologies through the application layer can be accomplished by IoT.

Reference [106] presents a four-layer architecture that consists of the application layer, cloud layer, network layer, and device layer. The device layer consists of two sublayers, a thin layer and a gateway layer. Thing layer contains various type of sensors, tags, actuators, and smart meters. The gateway layer contains communication modules, microcontrollers, storage, and local display. The services are provided to end user by application layer, and it includes dynamic pricing, demand response management, and energy management. Wang et al. [107] also proposed a fourlayer architecture for IoT-based SG systems. It is comprised of the master station layer, remote communication layer, fieldwork layer, and terminal layer. In this architecture, the master station layer relates to the application layer of the IoT, the remote communication layer relates to the network layer, and the field network and terminal layers are related to the perception layer of the IoT. In [108], the authors reviewed the four-layer and three-layer models. They also proposed a four-layer model that consists of the social layer at the top followed by the perception layer, network layer, and application layer. The IoT applications are regulated by the social layer.

A five-layer architecture model named "Smart Grid Architecture Model (SGRAM)" is the outcome of EU Mandate M/490's architecture group [109]. It is comprised of component, communication, information, function, and business layers as given in Table 5. These are defined as interoperability layers [110]. Every interoperability layer consists of SG plane that deals with information management zones and electrical domains. The main objective of this model is the interaction of various domains with each other over information management zones.

\section{Integration of Edge Computing with IoT- Enabled Smart Grid}

The smart grid is a pioneering technology that is transforming the conventional grids so that utility costs and global warming could be turned down using smart appliances, renewable energy resources, and smart meters. The IoT-enabled smart grid has already been largely adopted in terms of information sensing, processing, and transmission. Traditional cloud computing has not been able to fulfil the needs of IoT data processing because cloud servers are located geographically in distant areas that need multihop communication. The communication delay is not acceptable in some real-time applications. IoT devices transmit raw data to the clouds that impose a massive load on the communication network. Therefore, response time and latency 
TABLE 5: Architecture layers for IoT-based SG.

\begin{tabular}{|c|c|c|c|c|c|}
\hline & {$[100-105]$} & [106] & [107] & [108] & {$[109,110]$} \\
\hline Layer 5 & & & & & Business \\
\hline Layer 4 & & Application & Master station system & Social & Function \\
\hline Layer 3 & Application & Cloud management & Remote communication & Application & Information \\
\hline Layer 2 & Network & Network & Field work & Network & Communication \\
\hline Layer 1 & Perception & Perception & Terminal & Perception & Component \\
\hline
\end{tabular}

become unreliable. The data gathered by IoT contain private or sensitive information related to the user, so if the raw data are directly sent to the public cloud, then the third party can approach that data and it will raise security and privacy concern. To address these issues, EC performs data processing at the edge of the IoT system. EC reduces the network load as well as the latency for real-time applications. EC is considered a favorable solution to overcome the drawbacks of cloud computing in the smart grid as it provides storage resources and intensive computation at the edge of the network [111].

5.1. Framework of EC-IoT-Enabled SG. An EC system for IoT-enabled $S G$ is proposed in [12] to overcome the drawbacks of the present cloud computing paradigm. The paper proposes an EC hardware and software framework for SG which is comprised of 5 layers (device layer, network layer, data layer, application layer, and cloud computing layer). It is presented in three layers: the device layer, the edge layer, and the cloud layer. The device layer is the first layer that functions as a communication bridge between SG devices, and it consists of IoT objects like actuators, controllers, and sensors that monitor equipment, smart meters, smart appliances, services, or activities in SG operation. The IoT devices can perform preprocessing on the data, but these devices are not capable of fully processing the data because of their limited computation and memory. Therefore, they offload the rest of the computation to the upper layers. Normally, a wireless standard is applied in IoT environments such as Bluetooth, WiFi, ZigBee, 4G/5G, and so on [112].

Edge is the central layer in the framework. It is situated at the edge of the network and comprises a large number of edge nodes. In this layer, the data produced in the device layer are filtered and preprocessed and then transferred to the cloud. Various edge nodes at different locations in the SG system send real-time data to the edge server where necessary computation is performed and the results are sent to the central data centre in the cloud. Edge gateways are computers with larger capacities that perform the function of intersection among the IoT layer devices and the cloud. This layer also includes network management, computing management, and memory management modules [12]. The edge computing devices ensure the secure access of various objects in the device layer and maintain themselves and the cloud computing layer to work collectively through application program interfaces (APIs). As edge servers are deployed close to the users, by providing storage and computational resources at the edge, EC can reduce the latency for end devices and can provide a real-time response for end-user applications [113].
Cloud computing is the third layer in the framework. EC does not suggest that cloud computing will be forbidden; it is still a key element of hierarchical structure for IoT and is a combined storage and computing platform. It is comprised of storage devices and various high-performance servers that can store and process large-scale data and provide various application services. It is in charge of permanent data storage and computational analysis of datasets. This layer receives summarized data from each edge node and carries out a global analysis of the received data. Moreover, the cloud computing layer forwards policies to the edge layer to enhance the quality of latency-sensitive services [114].

The purpose of EC in SG is to compensate for the highlatency, drastic network energy consumption of the cloud and to provide security and real-time response to end users. This framework can solve the problems of real-time requirements, the heterogeneous connection of massive data, and intelligence in the SG system.

5.2. EC-IoT-Enabled SG Requirements. To turn the vision of EC-enabled SG into reality, some core requirements are outlined and explained. These requirements include compatibility, sustainability, reliability and scalability, resource management, flexibility, security, and privacy.

5.2.1. Compatibility. Compatibility is the ability of various systems to understand and make use of each other functions. Compatibility or interoperability is one of the critical requirements in the design of EC-IoT-enabled SG systems. The challenge of compatibility in EC-IoT-enabled SG arises because of a large number of heterogeneous devices using different protocols. To empower smooth operation, EC frameworks must be able to deliver support for compatibility as shown in Figure 3. It can be implemented in SGs by opensource frameworks and utilizing interoperable interfaces.

5.2.2. Sustainability. Another important requirement for designing EC infrastructure for SG is sustainability. It is referred to as the utilization of renewable energy resources and energy-efficient designs to minimize the universal carbon footprint. The three major aspects that are connected to the sustainability of EC-IoT-enabled SG are energy-efficient design $[115,116]$, energy harvesting [117-119], and use of renewable energy sources [120]. There are three important characteristics of energy-efficient design, such as energy-efficient resource management, energy-efficient hardware, and energy-efficient software. The function of energy harvesting is 

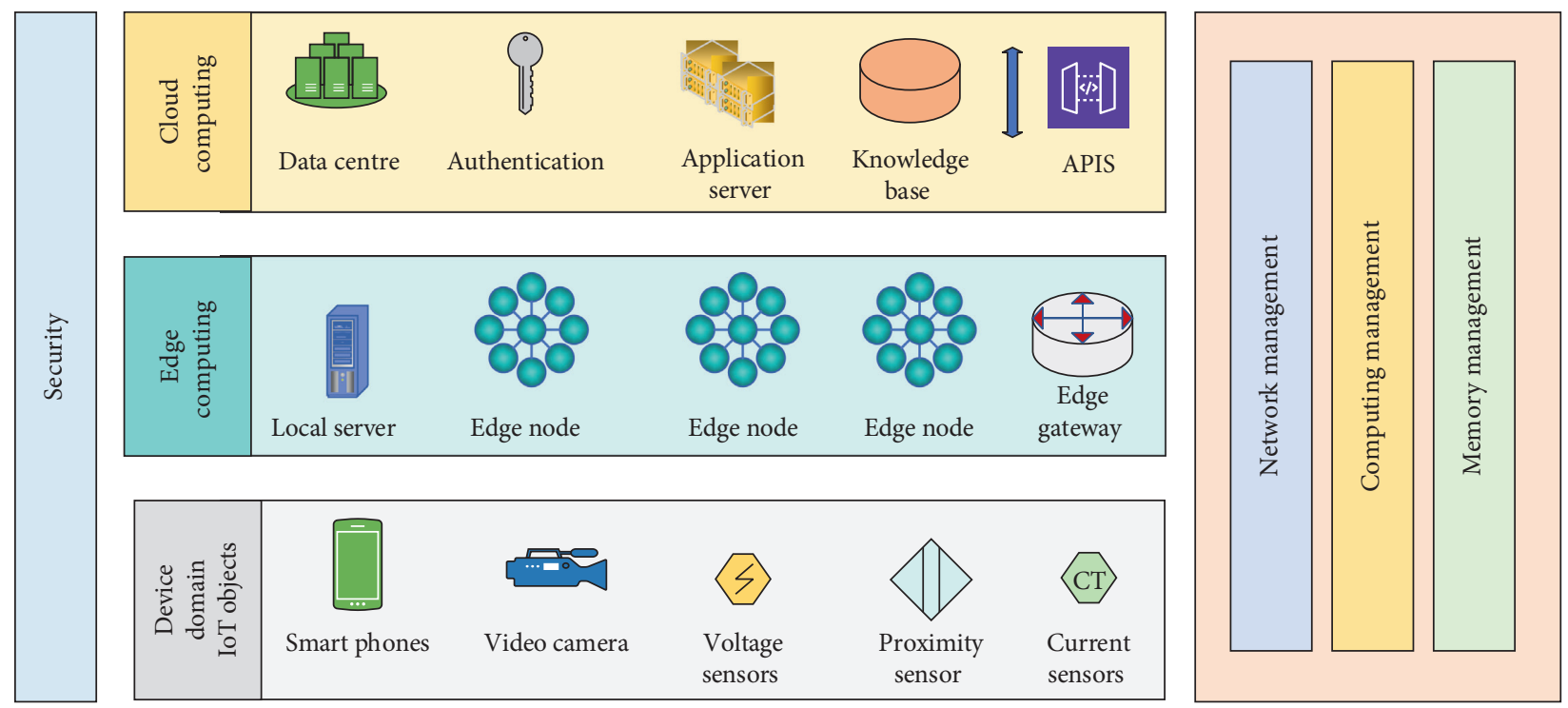

Figure 3: Framework of EC-IoT-enabled SG.

to extract the energy from external sources to operate small devices such as wearable devices and sensors.

5.2.3. Reliability and Scalability. In the smart grid, there will be a large number of sensors, actuators, and other smart devices, due to which data traffic will be increased exceptionally. EC gives a solution to enable latency-sensitive and resource-intensive SG applications. To establish a scalable EC infrastructure, we have to contemplate reliable performance for the increasing number of smart devices, diverse networks, and end users [121]. Scalability in EC infrastructure can be obtained by adding the new service providing points. Scalable hardware and scalable software are required for the inclusion of new serving points. On the other hand, elevated issues of reliability in EC infrastructure can be raised due to its hardware/software failures and dynamic nature.

5.2.4. Resource Management. For efficient management of computation, storage, and communication resources among various players in SG for the optimization of overall system performance, we must come up with resource management schemes that contemplate latency [122], energy [123-125], user-defined utility [126], and capacity of traffic handling [127] to optimize comprehensive system performance.

5.2.5. Flexibility. Flexibility in EC avoids underallocation and overallocation of computing resources. It can be recognized in SG infrastructure by delivering flexible services based on the resources which can shrink and grow dynamically according to the demand [128, 129]. For flexibility, it is necessary to adequately predict user demand using edge service providers before assigning computational resources, like storage, bandwidth, and processing power. EC-based smart devices should be flexible. Reference [130] presents a protective scheme that is based on automatic computing and reinforcement learning concepts. Techniques of autoscaling can be horizontal, vertical, or hybrid [131, 132]. Horizontal scaling is to resize the resource allocation between a cluster of nodes cluster, whereas vertical scaling is to resize the resource allocation at the same node [133]. The hybrid approach integrates both horizontal and vertical schemes [134].

5.2.6. Security and Privacy. There are two major aspects of security in EC: physical security and cybersecurity of devices.

Cybersecurity is related to the protection of data, network, and computing infrastructure from attacks. Privacy defines some set of rules that provide protection levels. Restrictions are placed by privacy on the authorization of data access. At the centre of EC paradigms, there are various technology enablers, e.g., wireless networks, virtualization, and peer-to-peer and distributed systems. Figure 4 shows the EC-IoT-enabled SG requirements. Therefore, physical security of edge devices is mandatory and it is also very challenging because edge nodes have distributed nature. Powerful cryptographic techniques can also be utilized to prevent attacks from the attacker.

\section{Open Research Issues and Challenges}

Security mechanisms in EC may be centralized or decentralized. Centralized security needs uninterrupted accessibility of centralized infrastructure. It has the edge of easier management, but it is at risk of failure if the centralized server is exposed to any attack. While a mechanism of decentralized security has the benefits of less vulnerability to attacks and reduction in delay, this mechanism is more complex than the centralized one because of its distributed nature.

EC-IoT-based SG systems will operate in unfavorable conditions, like power transmission lines monitoring. So, it will be helpful to inspect requirements for hybrid communication technologies and coverage of signals in 


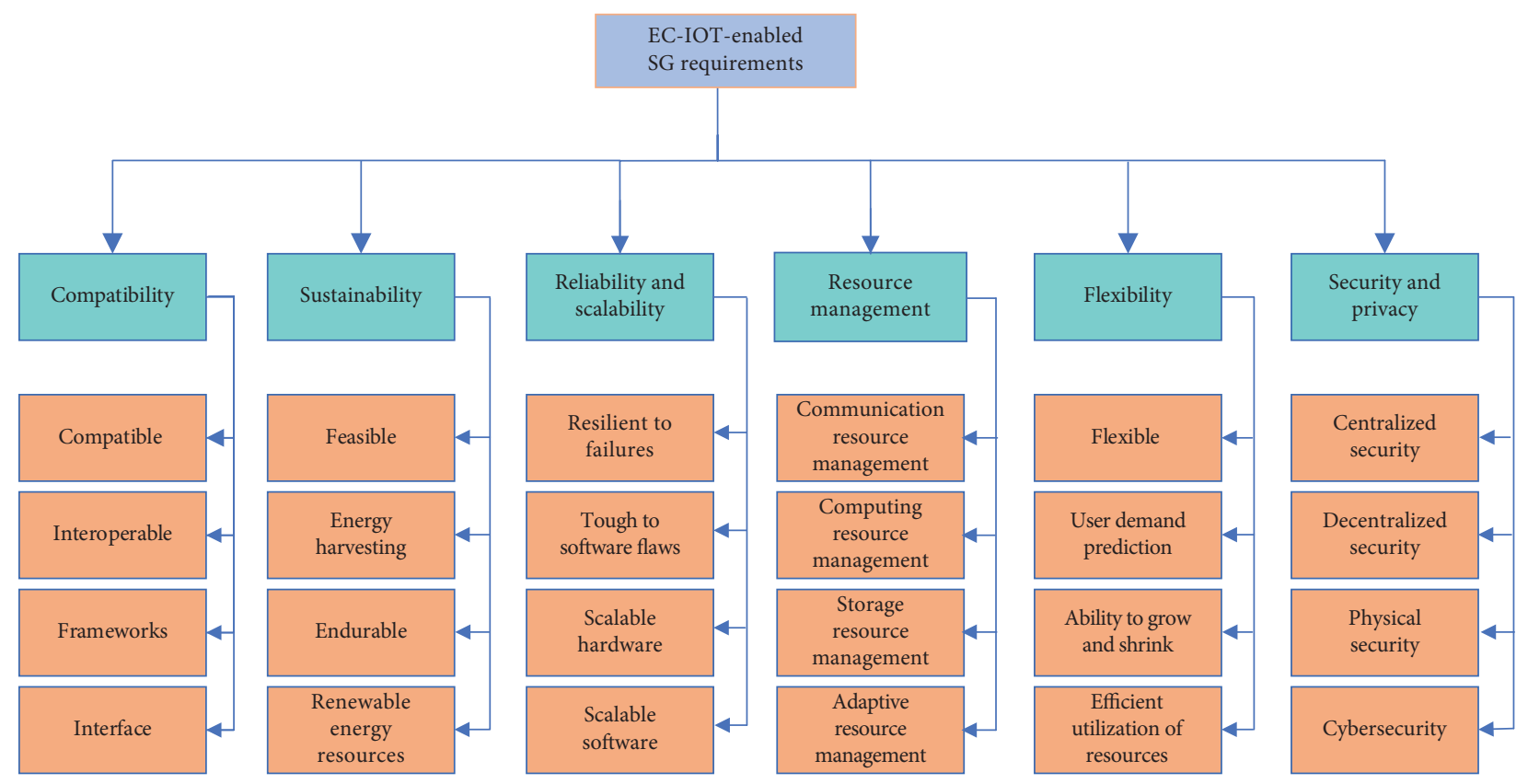

Figure 4: EC-IoT-enabled SG requirements.

unfavorable environmental conditions, in terms of compatibility, availability, and reliability. IoT sensors and end devices run on batteries in various applications of SG systems. For example, various sensors, backbone nodes, and video cameras are installed on the transmission lines and transmission towers for the monitoring of power transmission. Normally, these devices run on batteries. Therefore, the source of energy for power consumption is a critical problem. To solve this issue, efficient resources of energy storage and energy generation devices that are coupled with energy harvesting by using energy conversion should be designed. Information and communication networks are critical for the transmission and cooperation of edge servers and IoT devices. EC-IoT-based SG systems use a combination of different communication networks at various stages in the same operation. The communication path proceeds through several terminals, devices, local network, gateways, edge servers, and finally to the cloud, utilizing different protocols every time. This means that SG systems require rigid network support despite multiple network providers and fragmented communication phases.

The EC should be configurable and information aware to take on massive packet traffic. The utilization of big data technology is feasible to increase the self-organizing abilities in EC. Edge devices collect a large volume of data and transmit them to the cloud; it puts a huge burden on the front haul/backhaul. To handle this, preexecution of data mining in EC can be performed. Moreover, the computing of incomplete and uncertain data is a big problem that needs advanced data mining algorithms. Harvesting of energy can also be performed from eco-friendly resources such as thermal, solar, and wind. Renewable energy resources involve geothermal, biomass, and wind energy. Algorithms of resource management should be adaptive, and they should perform management according to the nature of applications to enable cost-efficient operation for a better quality of service. To handle system failures, EC infrastructure must be used within SGs that are resilient to software/hardware failures.

\section{Conclusion}

SG is an underlying technology that will provide significant changes to the present power grid, energy resources, and consumer lives. It will solve the problems of unidirectional energy flow, growing energy demand, energy wastage, security, and reliability in the conventional power grid. SG consists of a large number of data sources and sensors that continuously gather high-resolution data. Handling this massive amount of data is a bottleneck for IoT and cloudcentric designs, particularly when latency-sensitive and real-time services exist. EC is envisioned as a provisioning solution to enable the cost-effective, efficient, and reliable two-way energy and information flow in SG because it provides computation and storage resources at the edge of the network. In this paper, we have presented a comprehensive review on EC-IoT-enabled SG. Framework for ECIoT-based SG is examined, and important requirements to implement the EC-IoT-based SG system are outlined. Finally, some critical issues and challenges faced in the implementation of EC-IoT-based SG systems are identified.

\section{Data Availability}

No data were used to support this study.

\section{Conflicts of Interest}

The authors declare that they have no conflicts of interest. 


\section{References}

[1] S. E. Collier, "The emerging enernet: convergence of the smart grid with the Internet of Things," IEEE Industry Applications Magazine, vol. 23, no. 2, pp. 12-16, 2016.

[2] R. Deng, Z. Yang, M. Y. Chow, and J. Chen, "A survey on demand response in smart grids: mathematical models and approaches," IEEE Transactions on Industrial Informatics, vol. 11, no. 3, pp. 570-582, 2015.

[3] R. Ma, H. H. Chen, Y. R. Huang, and W. Meng, "Smart grid communication: its challenges and opportunities," IEEE transactions on Smart Grid, vol. 4, no. 1, pp. 36-46, 2013.

[4] M. M. Rana, W. Xiang, and E. Wang, "Iot-based state estimation for microgrids," IEEE Internet of Things Journal, vol. 5, no. 2, pp. 1345-1346, 2018.

[5] B. N. Alhasnawi and B. H. Jasim, "Internet of Things (IoT) for smart grids: a comprehensive review," Journal of Xi'an University of Architecture \& Technology, vol. 11, no. 5, 2020.

[6] H. Sun, A. Nallanathan, B. Tan, J. S. Thompson, J. Jiang, and H. V. Poor, "Relaying technologies for smart grid communications," IEEE Wireless Communications, vol. 19, no. 6, pp. 52-59, 2012.

[7] M. Yigit, V. C. Gungor, and S. Baktir, "Cloud computing for smart grid applications," Computer Networks, vol. 70, pp. 312-329, 2014.

[8] E. Yaacoub and A. Abu-Dayya, "Automatic meter reading in the smart grid using contention based random access over the free cellular spectrum," Computer Networks, vol. 59, pp. 171-183, 2014.

[9] W. Meng, R. Ma, and H. H. Chen, "Smart grid neighborhood area networks: a survey," IEEE Network, vol. 28, no. 1, pp. 24-32, 2014.

[10] F. Samie, L. Bauer, and J. Henkel, "Iot technologies for embedded computing: a survey," in Proceedings of 2016 International Conference on Hard- ware/Software Codesign and System Synthesis (CODES+ ISSS), pp. 1-10, IEEE, Pittsburgh, PA, USA, October 2016.

[11] S. S. Chen, H. H. Xu, D. D. Liu, B. B. Hu, and H. H. Wang, "A vision of IOT: applications, challenges, and opportunities with China perspective," IEEE Internet of Things journal, vol. 1, no. 4, pp. 349-359, 2014.

[12] S. Chen, H. Wen, J. Wu et al., "Internet of Things based smart grids supported by intelligent edge computing," IEEE Access, vol. 7, Article ID 74089, 2019.

[13] H. Abd ul Muqeet, "Optimal operation of the campus microgrid considering the resource uncertainty and demand response schemes," Mathematical Problems in Engineering, vol. 2021, Article ID 5569701, 18 pages, 2021.

[14] J. Golrokh and A. Hasan, "A comparison of machine learning clustering algorithms based on the DEA optimization approach for pharmaceutical companies in developing countries," ENG Transactions, vol. 1, pp. 1-8, 2020.

[15] N. A. Golilarz, A. Addeh, H. Gao et al., "A new automatic method for control chart patterns recognition based on ConvNet and Harris hawks meta heuristic optimization algorithm," IEEE Access, vol. 7, pp. 149398-149405, 2019.

[16] A. Addeh and M. Iri, "Brain tumor type classification using deep features of MRI images and optimized RBFNN," ENG Transactions, vol. 2, no. 1, pp. 1-7, 2021.

[17] J. Arshad, "Intelligent greenhouse monitoring and control scheme: an arrangement of sensors, raspberry Pi based embedded system and IoT platform," Indian Journal of Science and Technology, vol. 13, no. 27, pp. 2811-2822, 2020.
[18] J. Guo, H. Zheng, B. Li, and G.-Z. Fu, "A Bayesian approach for degradation analysis with individual differences," IEEE Access, vol. 7, Article ID 175033, 2019.

[19] J. Guo, H. Zheng, B. Li, and G. Z. Fu, "Bayesian hierarchical model-based information fusion for degradation analysis considering non-competing relationship," IEEE Access, vol. 7, Article ID 175222, 2019.

[20] W. Yu, F. Liang, X. He et al., "A survey on the edge computing for the Internet of Things," IEEE access, vol. 6, pp. 6900-6919, 2017.

[21] T. Taleb, K. Samdanis, B. Mada, H. Flinck, S. Dutta, and D. Sabella, "On multi-access edge computing: a survey of the emerging 5G network edge cloud architecture and orchestration," IEEE Communications Surveys \& Tutorials, vol. 19, no. 3, pp. 1657-1681, 2017.

[22] J. Moura and D. Hutchison, "Game theory for multi-access edge com- puting: survey, use cases, and future trends," IEEE Communications Surveys \& Tutorials, vol. 21, no. 1, pp. 260-288, 2018.

[23] P. Porambage, J. Okwuibe, M. Liyanage, M. Ylianttila, and T. Taleb, "Survey on multi-access edge computing for Internet of Things realization," IEEE Communications Surveys \& Tutorials, vol. 20, no. 4, pp. 2961-2991, 2018.

[24] S. Shahzadi, M. Iqbal, T. Dagiuklas, and Z. U. Qayyum, "Multi-access edge computing: open issues, challenges and future perspectives," Journal of Cloud Computing, vol. 6, no. 1, 2017.

[25] Y. Ai, M. Peng, and K. Zhang, "Edge computing technologies for Internet of Things: a primer," Digital Communications and Networks, vol. 4, no. 2, pp. 77-86, 2018.

[26] S. Wang, X. Zhang, Y. Zhang, L. Wang, J. Yang, and W. Wang, "A survey on mobile edge networks: convergence of computing, caching and communications," IEEE Access, vol. 5, pp. 6757-6779, 2017.

[27] Y. Mao, C. You, J. Zhang, K. Huang, and K. B. Letaief, “A survey on mobile edge computing: the communication perspective," IEEE Communications Surveys \& Tutorials, vol. 19, no. 4, pp. 2322-2358, 2017.

[28] R. Roman, J. Lopez, and M. Mambo, "Mobile edge computing, fog et al.: a survey and analysis of security threats and challenges," Future Generation Computer Systems, vol. 78, pp. 680-698, 2018.

[29] I. Afolabi, T. Taleb, K. Samdanis, A. Ksentini, and H. Flinck, "Network slicing and softwarization: a survey on principles, enabling technologies, and solutions," IEEE Communications Surveys \& Tutorials, vol. 20, no. 3, pp. 2429-2453, 2018.

[30] A. C. Baktir, A. Ozgovde, and C. Ersoy, "How can edge computing benefit from software-defined networking: a survey, use cases, and future directions," IEEE Communications Surveys \& Tutorials, vol. 19, no. 4, pp. 2359-2391, 2017.

[31] D. Sabella, A. Vaillant, P. Kuure, U. Rauschenbach, and F. Giust, "Mobile-edge computing architecture: the role of MEC in the Internet of Things," IEEE Consumer Electronics Magazine, vol. 5, no. 4, pp. 84-91, 2016.

[32] P. Mach and Z. Becvar, "Mobile edge computing: a survey on architecture and computation offloading," IEEE Communications Surveys \& Tutorials, vol. 19, no. 3, pp. 1628-1656, 2017.

[33] P. Garcia Lopez, A. Montresor, D. Epema et al., "Edgecentric computing: vision and challenges," ACM SIGCOMM Computer Communication Review, vol. 45, no. 5, pp. 37-42, 2015. 
[34] F. A. Turjman and M. Abujubbeh, "Iot-enabled smart grid via sm: an overview," Future Generation Computer Systems, vol. 96, pp. 579-590, 2019.

[35] S. Pawar and B. F. Momin, "Smart Electricity Meter Data Analytics: A Brief Review," in Proceedings of the 2017 IEEE Region 10 Symposium (TENSYMP), pp. 1-5, IEEE, Cochin, Kerala, India, July 2017.

[36] G. D. L. T. Parra, P. Rad, and K. K. R. Choo, "Implementation of deep packet inspection in smart grids and industrial Internet of Things: challenges and opportunities," Journal of Network and Computer Applications, vol. 135, pp. 32-46, 2019.

[37] G. Bedi, G. K. Venayagamoorthy, R. Singh, R. R. Brooks, and K. C. Wang, "Review of Internet of Things (IoT) in electric power and energy systems," IEEE Internet of Things Journal, vol. 5, no. 2, pp. 847-870, 2018.

[38] S. Jain, A. Paventhan, V. K. Chinnaiyan, V. Arnachalam, M. Pradish et al., "Survey on smart grid technologies-smart metering, Iot and Ems," in Proceedings of the 2014 IEEE Students' Conference on Electrical, Electronics and Computer Science, pp. 1-6, IEEE, Bhopal, India, March 2014.

[39] Q. Sun, H. Li, Z. Ma et al., "A comprehensive review of smart energy meters in intelligent energy networks," IEEE Internet of Things Journal, vol. 3, no. 4, pp. 464-479, 2015.

[40] M. B. Mollah, J. Zhao, D. Niyato et al., "Blockchain for future smart grid: a comprehensive survey," IEEE Internet of Things Journal, vol. 8, no. 1, pp. 18-43, 2020.

[41] S. M. Matinkhah and W. Shafik, "Smart Grid Empowered by 5g Technology," in Proceedings of the 2019 Smart Grid Conference (SGC), pp. 1-6, Tehran, Iran, December 2019.

[42] A. Ghosal and M. Conti, "Key management systems for smart grid advanced metering infrastructure: a survey," IEEE Communications Surveys \& Tutorials, vol. 21, no. 3, pp. 2831-2848, 2019.

[43] A. S. Musleh, G. Chen, and Z. Y. Dong, "A survey on the detection algorithms for false data injection attacks in smart grids," IEEE Transactions on Smart Grid, vol. 11, no. 3, pp. 2218-2234, 2020.

[44] P. Kumar, Y. Lin, G. Bai, A. Paverd, J. S. Dong, and A. Martin, "Smart grid metering networks: a survey on security, privacy and open research issues," IEEE Communications Surveys \& Tutorials, vol. 21, no. 3, pp. 2886-2927, 2019.

[45] I. Serban, S. Ce'spedes, C. Marinescu, C. A. Azurdia-Meza, J. S. Go'mez, and D. S. Hueichapan, "Communication requirements in microgrids: a practical survey," IEEE Access, vol. 8, Article ID 47694, 2020.

[46] B. Petersen, H. Bindner, B. Poulsen, and S. You, "Smart grid commu- nication comparison: distributed control middleware and serialization comparison for the Internet of Things," in Proceedings of the 2017 IEEE PES Innovative Smart Grid Technologies Conference Europe (ISGT-Europe), pp. 1-6, Turin, Italy, September 2017.

[47] Y. Saleem, N. Crespi, M. H. Rehmani, and R. Copeland, "Internet of Things-aided smart grid: technologies, architectures, applications, prototypes, and future research directions," IEEE Access, vol. 7, Article ID 62962, 2019.

[48] A. Gupta, A. Anpalagan, G. H. Carvalho, A. S. Khwaja, L. Guan, and I. Woungang, "Prevailing and Emerging Cyber Threats and Security Practices in Iot-Enabled Smart Grids: A survey," Journal of Network and Computer Applications, vol. 132, 2019.

[49] A. Alwarafy, K. A. Al-Thelaya, M. Abdallah, J. Schneider, and M. Hamdi, "A survey on security and privacy issues in edge computing- assisted internet of things," IEEE Internet of Things Journal, vol. 8, no. 6, 2020.

[50] X. Chen, L. Sun, H. Zhu, Y. Zhen, and H. Chen, "Application of Internet of Things in power-line monitoring," in Proceedings of the 2012 International Conference on Cyber-Enabled Distributed Computing and Knowledge Discovery, pp. 423-426, IEEE, Sanya, China, October 2012.

[51] J. Gubbi, R. Buyya, S. Marusic, and M. Palaniswami, "Internet of Things (IoT): a vision, architectural elements, and future directions," Future Generation Computer Systems, vol. 29, no. 7, pp. 1645-1660, 2013.

[52] Y. Lu and L. Da Xu, "Internet of Things (IoT) cybersecurity research: a review of current research topics," IEEE Internet of Things Journal, vol. 6, no. 2, pp. 2103-2115, 2018.

[53] A. Colakovic' and M. Hadzialic', "Internet of Things (IoT): a review of enabling technologies, challenges, and open research issues," Computer Networks, vol. 144, pp. 17-39, 2018.

[54] S. Balaji, K. Nathani, and R. Santhakumar, "IoT technology, applications and challenges: a contemporary survey," Wireless Personal Communications, vol. 108, no. 1, pp. 363-388, 2019.

[55] M. Bembe, A. A. Mahfouz, M. Masonta, and T. Ngqondi, "A survey on low-power wide area networks for iot applications," Telecommunication Systems, vol. 71, no. 2, pp. 249-274, 2019.

[56] M. Z. Hasan, H. A. Rizzo, and F. A. Turjman, "A survey on multipath routing protocols for qos assurances in real-time wireless multimedia sensor networks," IEEE Communications Surveys \& Tutorials, vol. 19, no. 3, pp. 1424-1456, 2017.

[57] R. Ahmed, A. K. Malviya, M. J. Kaur, and V. P. Mishra, "Compre- hensive survey of key technologies enabling 5giot," in Proceedings of the 2nd International Conference on Advanced Computing and Software Engineering (ICACSE), Sultanpur, India, February 2019.

[58] A. Adeel, M. Gogate, S. Farooq et al., "A survey on the role of wireless sensor networks and iot in disaster management," in Geological Disaster Monitoring Based on Sensor Networks, pp. 57-66, Springer Natural Hazards, The Gateway, Singapore, 2019.

[59] J. Yin, Z. Yang, H. Cao, T. Liu, Z. Zhou, and C. Wu, "A survey on Bluetooth 5.0 and mesh," ACM Transactions on Sensor Networks, vol. 15, no. 3, pp. 1-29, 2019.

[60] J. Khan, J. P. Li, A. U. Haq et al., "Efficient secure surveilance on smart healthcare IoT system through cosine-transform encryption," Journal of Intelligent and Fuzzy Systems, vol. 40, no. 1, pp. 1417-1442, 2021.

[61] A. Zanella, N. Bui, A. Castellani, L. Vangelista, and M. Zorzi, "Internet of Things for smart cities," IEEE Internet of Things journal, vol. 1, no. 1, pp. 22-32, 2014.

[62] H. Habibzadeh, K. Dinesh, O. Rajabi Shishvan, A. BoggioDandry, G. Sharma, and T. Soyata, "A survey of healthcare Internet of Things (hiot): a clinical perspective," IEEE Internet of Things Journal, vol. 7, no. 1, pp. 53-71, 2020.

[63] H. Tran-Dang, N. Krommenacker, P. Charpentier, and D. S. Kim, "Towards the Internet of Things for physical internet: perspectives and challenges," IEEE Internet of Things Journal, 2020

[64] S. Weisong, S. Hui, C. Jie, Z. Quan, and L. Wei, "Edge computing-an emerging computing model for the internet of everything era," Journal of Computer Research and Development, vol. 54, no. 5, p. 907, 2017.

[65] S. Weisong, Z. Xingzhou, W. Yifan, and Z. Qingyang, "Edge com- puting: state-of-the-art and future directions," Journal 
of Computer Research and Development, vol. 56, no. 1, p. 69, 2019.

[66] W. Shi, J. Cao, Q. Zhang, Y. Li, and L. Xu, "Edge computing: vision and challenges," IEEE Internet of Things journal, vol. 3, no. 5, pp. 637-646, 2016.

[67] M. Satyanarayanan, "The emergence of edge computing," Computer, vol. 50, no. 1, pp. 30-39, 2017.

[68] K. Cao, Y. Liu, G. Meng, and Q. Sun, “An overview on edge computing research," IEEE Access, vol. 8, Article ID 85714, 2020.

[69] X. Sun and N. Ansari, "EdgeIoT: mobile edge computing for the Internet of Things," IEEE Communications Magazine, vol. 54, no. 12, pp. 22-29, 2016.

[70] C. Mouradian, D. Naboulsi, S. Yangui, R. H. Glitho, M. J. Morrow, and P. A. Polakos, "A comprehensive survey on fog computing: state-of-the-art and research challenges," IEEE Communications Surveys \& Tutorials, vol. 20, no. 1, pp. 416-464, 2018.

[71] J. Kang, R. Yu, X. Huang, and Y. Zhang, "Privacy-preserved pseudonym scheme for fog computing supported internet of vehicles," IEEE Transactions on Intelligent Transportation Systems, vol. 19, no. 8, pp. 2627-2637, 2018.

[72] P. Hu and W. Chen, "Software-defined edge computing (sdec): prin- ciples, open system architecture and challenges," in Proceedings of the 2019 IEEE SmartWorld, Ubiquitous Intelligence Computing, Advanced Trusted Computing, Scalable Computing Communications, Cloud Big Data Computing, Internet of People and Smart City Innovation (Smart- World/SCALCOM/UIC/ATC/CBDCom/IOP/ SCI), pp. 8-16, Leicester, UK, August 2019.

[73] H. Zhang, Q. Li, Y. Qi, J. Hou, and Y. Liu, "The research reviewed of edge network active safety defense technology," in Proceedings of the 2019 IEEE Fifth International Conference on Big Data Computing Service and Applications (BigDataService), pp. 242-246, Newark, CA, USA, April 2019.

[74] F. Bonomi, R. Milito, J. Zhu, and S. Addepalli, "Fog computing and its role in the Internet of Things," in Proceedings of the First Edition of the MCC Workshop on Mobile Cloud Computing, pp. 13-16, New York, NY, USA, August 2012.

[75] M. Gusev and S. Dustdar, "Going back to the roots-the evolution of edge computing, an IoT perspective," IEEE Internet Computing, vol. 22, no. 2, pp. 5-15, 2018.

[76] C. Li, Y. Xue, J. Wang, W. Zhang, and T. Li, "Edge-oriented computing paradigms," ACM Computing Surveys, vol. 51, no. 2, pp. 1-34, 2018.

[77] M. Chiang, S. Ha, F. Risso, T. Zhang, and I. C. Lin, "Clarifying fog computing and networking: 10 questions and answers," IEEE Communications Magazine, vol. 55, no. 4, pp. 18-20, 2017.

[78] P. Neto, "Demystifying Cloud Computing," in Proceedings of the Doctoral Symposium on Informatics Engineering, vol. 24, pp. 16-21, Porto, Portugal, 2011.

[79] F. Liu, J. Tong, J. Mao et al., Nist Cloud Computing Reference Architecture, NIST Special Publication, Gaithersburg, MD, USA, 2011.

[80] K. Saharan and A. Kumar, "Fog in comparison to cloud: a survey," International Journal of Computer Application, vol. 122, no. 3, 2015.

[81] T. H. Luan, L. Gao, Z. Li, Y. Xiang, and L. Sun, "Fog computing: focusing on mobile users at the edge," CoRR, http://arxiv.org/abs/1502.01815, 2015.

[82] M. Yun and B. Yuxin, "Research on the architecture and key technology of Internet of Things (IoT) applied on smart grid," in Proceedings of the 2010 International Conference on Advances in Energy Engineering, pp. 69-72, IEEE, Beijing, China, June 2010.

[83] A. Al-Fuqaha, M. Guizani, M. Mohammadi, M. Aledhari, and M. Ayyash, "Internet of Things: a survey on enabling technologies, protocols, and applications," IEEE Communications Surveys \& Tutorials, vol. 17, no. 4, pp. 2347-2376, 2015.

[84] I. Yaqoob, E. Ahmed, I. A. T. Hashem et al., "Internet of Things architecture: recent ad- vances, taxonomy, requirements, and open challenges," IEEE wireless communications, vol. 24, no. 3, pp. 10-16, 2017.

[85] M. A. Zaveri, S. K. Pandey, and J. S. Kumar, "Collaborative service oriented smart grid using the Internet of Things," in Proceedings of the 2016 International Conference On Communication And Signal Processing (ICCSP), pp. 1716-1722, Melmaruvathur, India, April 2016.

[86] K. Samarakoon, J. Ekanayake, and N. Jenkins, "Reporting available demand response," IEEE Transactions on Smart Grid, vol. 4, no. 4, pp. 1842-1851, 2013.

[87] W. S. wen, "Research on the key technologies of iot applied on smart grid," in Proceedings of the 2011 International Conference On Electronics, Communica- Tions and Control (ICECC), pp. 2809-2812, Ningbo, China, September 2011.

[88] A. Basit, G. A. S. Sidhu, A. Mahmood, and F. Gao, "Efficient and autonomous energy management techniques for the future smart homes," IEEE Transactions on Smart Grid, vol. 8, no. 2, pp. 917-926, 2017.

[89] N. Raza, M. Q. Akbar, A. A. Soofi, and S. Akbar, "Study of smart grid communication network architectures and technologies," Journal of Computer and Communications, vol. 7, no. 3, pp. 19-29, 2019.

[90] S. Ahmed, T. M. Gondal, M. Adil, S. A. Malik, and R. Qureshi, "A survey on communication technologies in smart grid," in Proceedings of the 2019 IEEE PES GTD Grand International Conference and Exposition Asia (GTD Asia), pp. 7-12, Bangkok, Thailand, March 2019.

[91] H. Sharma, N. Kumar, and B. K. Panigrahi, "Physical layer security of ami data transmission in smart grid environment," in Proceedings of the 2019 IEEE Globecom Workshops (GC Wkshps), pp. 1-6, Waikoloa, HI, USA, December 2019.

[92] R. Morello, C. De Capua, G. Fulco, and S. C. Mukhopadhyay, "A smart power meter to monitor energy flow in smart grids: the role of advanced sensing and iot in the electric grid of the future," IEEE Sensors Journal, vol. 17, no. 23, pp. 7828-7837, 2017.

[93] C. H. Lo and N. Ansari, "The progressive smart grid system from both power and communications aspects," IEEE Communications Surveys \& Tutorials, vol. 14, no. 3, pp. 799-821, 2011.

[94] D. N. Molokomme, C. S. Chabalala, and P. N. Bokoro, "A review of cognitive radio smart grid communication infrastructure systems," Energies, vol. 13, no. 12, 2020.

[95] V. C. Gungor, D. Sahin, T. Kocak et al., "A survey on smart grid potential applications and communication requirements," IEEE Transactions on Industrial Informatics, vol. 9, no. 1, pp. 28-42, 2013.

[96] Y. Ding, X. Li, Y.-C. Tian, G. Ledwich, Y. Mishra, and C. Zhou, "Generating scale-free topology for wireless neighborhood area networks in smart grid," IEEE Transactions on Smart Grid, vol. 10, no. 4, pp. 4245-4252, 2019.

[97] Y. Ding, Y.-C. Tian, X. Li, Y. Mishra, G. Ledwich, and C. Zhou, "Constrained broadcast with minimized latency in neighborhood area networks of smart grid," IEEE 
Transactions on Industrial Informatics, vol. 16, no. 1, pp. 309-318, 2020.

[98] V. Terzija, G. Valverde, D. Deyu Cai et al., "Wide-area monitoring, protection, and control of future electric power networks," Proceedings of the IEEE, vol. 99, no. 1, pp. 80-93, 2011.

[99] R. Mahadevan, S. H. Kadati, S. Chandrasekhar, and S. Singandhupe, "Satellite communications for rural smart grid," Journal of Communications, vol. 2012, pp. 19-30, 2012.

[100] J. Lloret, J. Tomas, A. Canovas, and L. Parra, "An integrated IoT architecture for smart metering," IEEE Communications Magazine, vol. 54, no. 12, pp. 50-57, 2016.

[101] C. Wang, X. Li, Y. Liu, and H. Wang, "The research on development direction and points in iot in China power grid," in Proceedings of the 2014 International Conference on Information Science, Electronics and Electrical Engineering, pp. 245-248, IEEE, Sapporo, Japan, April 2014.

[102] X. Chen, J. Liu, X. Li, L. Sun, and Y. Zhen, "Integration of Iot with smart grid," in Proceedings of the IET International Conference on Communication Technology and Application (ICCTA 2011), Beijing, China, October 2011.

[103] J. Liu, X. Li, X. Chen, Y. Zhen, and L. Zeng, “Applications of Internet of Things on smart grid in China," in Proceedings of the 13th International Conference on Advanced Communication Technology (ICACT2011), pp. 13-17, Gangwon, Republic of Korea, February 2011.

[104] Q. Ou, Y. Zhen, X. Li, Y. Zhang, and L. Zeng, "Application of Internet of Things in smart grid power transmission," in Proceedings of the 2012 Third FTRA International Conference on Mobile, Ubiquitous, and Intelligent Computing, pp. 96100, Vancouver, Canada, June 2012.

[105] Y. E. Song, Y. Liu, S. Fang, and S. Zhang, "Research on applications of the Internet of Things in the smart grid," in Proceedings of the 2015 7th International Conference on Intelligent Human-Machine Systems and Cybernetics, pp. 178-181, Hangzhou, China, August 2015.

[106] S. K. Viswanath, C. Yuen, W. Tushar et al., "System design of the Internet of Things for residential smart grid," IEEE Wireless Communications, vol. 23, no. 5, pp. 90-98, 2016.

[107] Y. Wang, W. Lin, T. Zhang, and Y. Ma, "Research on application and security protection of Internet of Things in smart grid," in Proceeding of the IET International Conference on Information Science and Control Engineering 2012 (ICISCE 2012), Shenzhen, China, December-2012.

[108] H. Ning and S. Hu, "Technology classification, industry, and education for future Internet of Things," International Journal of Communication Systems, vol. 25, no. 9, pp. 1230-1241, 2012.

[109] J. Bruinenberg, L. Colton, E. Darmois et al., "Cen-cenelecetsi smart grid coordination group: smart grid reference architecture. cen, cenelec," Technical Report, ETSI, Technical Report, Sophia Antipolis, France, 2012.

[110] J. Trefke, S. Rohjans, M. Uslar, S. Lehnhoff, L. Nordstro"m, and A. Saleem, "Smart grid architecture model use case management in a large european smart grid project," in Proceedings of the IEEE PES ISGT Europe 2013, pp. 1-5, IEEE, Lyngby, Denmark, October-2013.

[111] T. X. Tran, A. Hajisami, P. Pandey, and D. Pompili, "Collaborative mobile edge computing in $5 \mathrm{G}$ networks: new paradigms, scenarios, and challenges," IEEE Communications Magazine, vol. 55, no. 4, pp. 54-61, 2017.

[112] C.-H. Ke, S.-Y. Hsieh, T.-C. Lin, and T.-H. Ho, "Efficiency network construction of advanced metering infrastructure using Zigbee," IEEE Transactions on Mobile Computing, vol. 18, no. 4, pp. 801-813, 2019.

[113] Y. Liu, M. Peng, G. Shou, Y. Chen, and S. Chen, "Toward edge intelligence: multiaccess edge computing for $5 \mathrm{G}$ and internet of things," IEEE Internet of Things Journal, vol. 7, no. 8, pp. 6722-6747, 2020.

[114] D. Li, "Edge computing, a compensation method for cloud computing on smart grid," Thesis, UNSW, Sydney, Sydney, Australia, 2019.

[115] S. H. Kim, S. Park, M. Chen, and C. H. Youn, “An optimal pricing scheme for the energy-efficient mobile edge computation offloading with ofdma," IEEE Communications Letters, vol. 22, no. 9, pp. 1922-1925, 2018

[116] X. Chen, L. Pu, L. Gao, W. Wu, and D. Wu, "Exploiting massive $\mathrm{d} 2 \mathrm{~d}$ collaboration for energy-efficient mobile edge computing," IEEE Wireless communications, vol. 24, no. 4, pp. 64-71, 2017.

[117] W. K. Lee, M. J. W. Schubert, B. Y. Ooi, and S. J. Q. Ho, "Multi-source energy harvesting and storage for floating wireless sensor network nodes with long range communication capability," IEEE Transactions on Industry Applications, vol. 54, no. 3, pp. 2606-2615, 2018.

[118] G. Qiao, S. Leng, K. Zhang, and K. Yang, "Joint deployment and mobility management of energy harvesting small cells in heterogeneous networks," IEEE Access, vol. 5, pp. 183-196, 2016.

[119] N. A. Chughtai, M. Ali, S. Qaisar, M. Imran, M. Naeem, and F. Qamar, "Energy efficient resource allocation for energy harvesting aided h-cran," IEEE Access, vol. 6, Article ID 43990, 2018.

[120] M. N. H. Nguyen, N. H. Tran, M. A. Islam, C. Pham, S. Ren, and C. S. Hong, "Fair sharing of backup power supply in multi-operator wireless cellular towers," IEEE Transactions on Wireless Communications, vol. 17, no. 3, pp. 2080-2093, 2018.

[121] E. Ahmed, A. Ahmed, I. Yaqoob et al., "Bringing computation closer toward the user network: is edge computing the solution?" IEEE Communications Magazine, vol. 55, no. 11, pp. 138-144, 2017.

[122] J. Ren, G. Yu, Y. Cai, and Y. He, "Latency optimization for resource allocation in mobile-edge computation offloading," IEEE Transactions on Wireless Communications, vol. 17, no. 8, pp. 5506-5519, 2018.

[123] S. E. Mahmoodi, K. Subbalakshmi, and V. Sagar, "Cloud offloading for multi-radio enabled mobile devices," in Proceedings of the 2015 IEEE International Conference on Communications (ICC), pp. 5473-5478, IEEE, London, UK, June 2015.

[124] C. You, K. Huang, and H. Chae, "Energy efficient mobile cloud computing powered by wireless energy transfer," IEEE Journal on Selected Areas in Communications, vol. 34, no. 5, pp. 1757-1771, 2016.

[125] J. Kwak, Y. Kim, J. Lee, and S. Chong, "Dream: dynamic resource and task allocation for energy minimization in mobile cloud systems," IEEE Journal on Selected Areas in Communications, vol. 33, no. 12, pp. 2510-2523, 2015.

[126] X. Lyu, H. Tian, C. Sengul, and P. Zhang, "Multiuser joint task offload- ing and resource optimization in proximate clouds," IEEE Transactions on Vehicular Technology, vol. 66, no. 4, pp. 3435-3447, 2016.

[127] M. Bouet and V. Conan, "Mobile edge computing resources optimization: a geo-clustering approach," IEEE Transactions on Network and Service Management, vol. 15, no. 2, pp. 787-796, 2018. 
[128] J. Xu and S. Ren, "Online learning for offloading and autoscaling in renewable-powered mobile edge computing," in Proceedings of 2016 IEEE Global Communications Conference (GLOBECOM), pp. 1-6, IEEE, Washington, DC, USA, December 2016.

[129] M. D. D. Assunção, A. D. S. Veith, and R. Buyya, "Distributed data stream processing and edge computing: a survey on resource elasticity and future directions," Journal of Network and Computer Applications, vol. 103, pp. 1-17, 2018.

[130] M. G. Arani, S. Jabbehdari, and M. A. Pourmina, “An autonomic resource provisioning approach for service-based cloud applications: a hybrid approach," Future Generation Computer Systems, vol. 78, pp. 191-210, 2018.

[131] S. Taherizadeh and V. Stankovski, "Auto-scaling applications in edge computing: taxonomy and challenges," in Proceedings of the Inter-National Conference on Big Data and Internet of Things, pp. 158-163, London, UK, December 2017.

[132] M. Caprolu, J. H. Fernandez, A. Alassi, and R. D. Pietro, "Increasing renewable generation feed-in capacity leveraging smart meters," in Proceedings of 2020 IEEE Green Energy and Smart Systems Conference (IGESSC), pp. 1-7, IEEE, Long Beach, CA, USA, November-2020.

[133] D. B. Avancini, J. J. P. C. Rodrigues, R. A. L. Rabêlo, A. K. Das, S. Kozlov, and P. Solic, "A new IoT-based smart energy meter for smart grids," International Journal of Energy Research, vol. 45, no. 1, pp. 189-202, 2021.

[134] K. Juanli and L. Li, "ZigBee-based architecture design of imperceptible smart home system," in Proceedings of the 9th International Conference on Computer Engineering and Networks, pp. 1003-1011, Springer, Singapore, 2021. 